Diagnosis

\section{Paediatric clinical decision support systems}

\section{P Ramnarayan, J Britto}

\section{Dreams come true?}

A computerised clinical decision support system (CDSS) is "a computer based tool using explicit knowledge to generate patient specific advice or interpretation". ${ }^{1}$ Our use of computers has been driven not only by the increasing need to manage large amounts of information, but also by the imperative to make evidence based and cost effective decisions on a daily basis. Furthermore, there is accumulating evidence to prove that computer aided medical tools address the growing information needs of the busy clinician ${ }^{3}$ and improve healthcare processes as well as patient outcomes. ${ }^{4}$ In turn, this has led to the rapid proliferation of a variety of CDSS. This leading article summarises the past, present, and future of such systems, with special emphasis on their role in paediatrics.

\section{THE PAST}

The concept of computerised decision support for medicine is not new. As far back as 1959, a pioneering article in Science described how computers might assist in the process of diagnosis. ${ }^{5}$ The familiar miniature device used by $\mathrm{Dr}$ McCoy to make diagnoses in the Star Trek series evolved out of a similar dream. This section describes some of the various approaches employed to develop previous CDSS.

In Warner's (1961) program for the diagnosis of congenital heart disease, data were drawn from 1035 patients referred for cardiac catheterisation. Given multiple clinical findings, a matrix of 33 different congenital heart diseases and 50 associated clinical findings was used to calculate the probability of a specific diagnosis. The diagnostic accuracy of this system matched that of three congenital heart disease experts. ${ }^{6}$ This was an early example of a system using Bayes' probabilistic theory. The eponymous theorem is reported to have been formulated by Reverend Thomas Bayes (1763) in an attempt to prove the existence of God, and calculates the updated probability of a disease, on the strength of additional evidence (say, a blood test result) and background information (prevalence of the disease). One of the most well researched Bayesian systems is the de Dombal system from Leeds, UK. This tool assisted in the diagnosis of abdominal pain, using data from 6000 patients. $^{7}$ Using this system in a multicentre trial showed improvements in diagnosis, decision making, and patient outcome. ${ }^{8}$ Other CDSS have used a rule based approach. In the MYCIN system used for the management of patients with infections, a set of "ifthen" rules was used to generate advice for physicians. ${ }^{9}$

A combination of Bayesian and rule based approaches was used in the MEDITEL-PEDS diagnostic system for paediatrics. ${ }^{10}$ In the DXplain medical diagnostic aid, scores for diseases were based on disease probabilities and heuristic methods ("rules of thumb"). ${ }^{11}$ AI/RHEUM provided assistance in the diagnosis of paediatric rheumatic diseases using a "criteria table" for each disease. ${ }^{12}$ Most of these systems have shown diagnostic accuracy rates of $80-90 \%{ }^{.12-14}$

Other approaches to the development of CDSS include the use of neural networks, and an artificial intelligence theory called "fuzzy logic". A recently
Table 1 Functions of CDSS and how they are used in clinical practice

\begin{tabular}{ll}
\hline Function & Example of routine use \\
\hline Alert & Clinical-laboratory systems highlighting abnormal values \\
Diagnosis & Producing a differential diagnosis for paediatric rheumatic diseases \\
Reminder & Reminding the clinician to schedule an immunisation visit \\
Suggestion & Suggesting adjustments to adjust mechanical ventilation \\
Interpretation & Paediatric electrocardiogram interpretation \\
Prediction & Predicting mortality from a Paediatric Index of Mortality (PIM) score \\
Critique & Reviewing total parenteral nutrition prescriptions \\
Assistance & Assisting selection of optimal antibiotic choices in neonatal infections \\
\hline
\end{tabular}

described neural network accurately distinguished between pathological and innocent heart murmurs in children when fed digital sound samples using an electronic stethoscope. ${ }^{15}$ A neonatal pulse oximeter that differentiated between "true" and "false" alarms provides an example of the use of "fuzzy logic". ${ }^{16}$

\section{THE PRESENT}

\section{Where do we use CDSS in routine} practice today?

Although the previous section mainly dealt with diagnostic support systems, decision support can be provided by any computer system that deals with clinical data or medical knowledge and delivers patient specific advice. Laboratory systems that flag abnormal values are examples of commonly used CDSS. NHS Direct, the national nurse led telephone triage system, is based on the use of computerised algorithms to provide decision support for a variety of clinical conditions. Table 1 summarises the various functions of CDSS and examples of how we use them in routine practice.

\section{Why do we need CDSS?}

Today, more than at any time in the past, clinicians are grappling with information overload. It is estimated that we use nearly 2 million pieces of information in our decision making and that biomedical knowledge is doubling every 20 years. ${ }^{17}$ We ask at least one clinical question during each encounter with a patient and fail to find answers to more than half of our questions. ${ }^{18}$

A potentially sick child in the current UK National Health Service (NHS) healthcare delivery model is likely to be first seen by relatively junior doctors at the apex of an "inverted pyramid of knowledge". Clinical knowledge and "wisdom" are concentrated among senior doctors at the base of this pyramid, often several steps removed from the patient. Such a systematic problem may be one reason why medical error is relatively common within the healthcare system. ${ }^{19}$ Errors and adverse events may be diagnostic, prescription based, or operative in nature; however, their collective impact might contribute to significant morbidity and economic loss. ${ }^{20}$

\section{ISABEL}

We have been closely involved in a novel project which aims to provide a free, web based decision support system for paediatrics and child health. ISABEL (http:// www.isabel.org.uk) is a product of the Isabel Medical Charity, ${ }^{20 a}$ founded as a consequence of a missed diagnosis in a 3 year old girl with chickenpox complicated by necrotising fasciitis. Using proprietary pattern recognition software called Autonomy (www.autonomy.com) to search standard paediatric textbooks, 
a differential diagnostic tool produces a list of up to 15 diagnoses to consider for any given set of clinical features. Further decision support is provided by text, annotated images, and practice guidelines specific to each diagnosis. A section entitled "experience" attempts to capture and highlight common clinical lessons learnt at various steps within the guidelines and at relevant points in the diagnostic process.

\section{Is it possible to evaluate the quality and the impact of CDSS?}

Evaluation of CDSS is a complex issue. There are many who feel that formal evaluation of their efficacy in randomised clinical trials is necessary, similar to any other intervention such as a new drug. ${ }^{21}$ Others believe that randomised trials may not be the best way to completely evaluate such tools and pose the question: "Can we imagine how randomised controlled trials would ensure the quality and safety of modern air travel ...? ${ }^{22}$ Nevertheless, rigorous evaluation of CDSS is imperative in a system driven by clinical governance as well as health economics. Table 2 summarises some of the considerations in the evaluation of CDSS.

\section{THE FUTURE}

Much work is being done on integrating CDSS into the clinical workflow; many current systems require the clinician to provide patient information to the CDSS either by typing or selecting from a range of options. However, the true potential of any CDSS will be realised only by linking it with an electronic patient record (EPR). This allows decision support to occur in the background, the system extracting patient details from the EPR and delivering patient specific advice to the user at appropriate points. The PRODIGY project integrated decision support for prescriptions into many NHS general practice EPR systems. ${ }^{23}$ Clearly, the future direction of new CDSSs in the UK is inextricably linked to that of the planned NHS EPR. Clinicians need to put in place systems now that will facilitate the easy integration of EPR with existing care pathways and local guidelines later.

Newer CDSS are also likely to utilise the structure and technology of the internet. As shown by the PubMed (http://www.ncbi.nlm.nih.gov/PubMed) initiative, it seems almost too easy to now bring together a diverse collection of information resources for a universal audience. The role of the internet and email in demystifying computers within the medical community will also play a key role in making decision support simple and acceptable to all users.

The future development of CDSS will also be influenced by medicolegal implications. $^{24}$ Patient confidentiality
Table 2 Steps in the evaluation of a CDSS and their utility

\begin{tabular}{|c|c|}
\hline Stage of evaluation & Questions answered \\
\hline Evaluation of structure & $\begin{array}{l}\text { Is the knowledge base of the CDSS "correct", is it well } \\
\text { represented and is it machine readable? }\end{array}$ \\
\hline $\begin{array}{l}\text { Evaluation of performance } \\
\text { Evaluation of clinical impact on: }\end{array}$ & Is the CDSS quick, accurate, and easy to use? \\
\hline Structural measures & Does it impact on the time spent, number of staff needed, etc \\
\hline Process measures & $\begin{array}{l}\text { Are appropriate tests and drugs being ordered? How accurate } \\
\text { are the clinical decisions taken? }\end{array}$ \\
\hline Outcome measures & Is patient morbidity and mortality affected by the CDSS? \\
\hline
\end{tabular}

and data protection will be major issues to bear in mind when delivering universal and easy EPR access through the web. ${ }^{25}$ Clinical risk management will influence the incorporation of CDSS into routine clinical use. The evaluation of CDSS will also be contentious: who should regulate or certify medical software? ${ }^{26}$

\section{CONCLUSIONS}

The paradigm shift in healthcare has ensured that CDSS are here to stay. These systems will work with clinicians, not instead of. Clinicians are also increasingly contributing to the development of these tools, rather than only acting as users. Newer technology, including the use of the internet, will revolutionise the way in which decision support is delivered in the future. Intelligent diagnostic tools reminiscent of the Star Trek series may not be far away, but Dr McCoy will need to stay.

\section{Arch Dis Child 2002;87:361-362}

\section{Authors' affiliations}

P Ramnarayan, J Britto, Imperial College School of Medicine, St Mary's Hospital, Norfolk Place, London, UK

Correspondence to: Dr J Britto, Imperial College School of Medicine, St Mary's Hospital, Norfolk Place, London W2 1PG, UK; j.britto@ic.ac.uk

\section{REFERENCES}

1 Wyatt J, Spiegelhalter D. Field trials of medical decision-aids: potential problems and solutions. In: Clayton P, ed. Proceedings of the 15th Symposium on Computer Applications in Medical Care, Washington 1991. New York: McGraw Hill, 1991:3-7.

2 Johnson KB, Feldman M. Medical informatics and pediatrics. Arch Pediatr Adolesc Med 1995;149:1371-80.

3 Osheroff JA, Bankowitz RA. Physicians' use of computer software in answering clinical questions. Bull Med Libr Assoc 1993;81:11-19.

4 Hunt DL, Haynes RB, Hanna SE, et al. Effects of computer-based clinical decision-support systems on physician performance and patient outcomes. JAMA 1998;280:1339-46.

5 Ledley RS, Lusted LB. Reasoning foundations of medical diagnosis. Science 1959;130:9-21.

6 Warner HR, Toronto AF, Veasey LG, et al. A mathematical approach to medical diagnosis: application to congenital heart disease. JAMA $1961 ; 177: 75-81$.
7 De Dombal FT, Leaper DJ, Staniland JR, et al. Computer-aided diagnosis of acute abdominal pain. BM 1972;2(5804):9-13

8 Adams ID, Chan M, Clifford PC, et al. Computer-aided diagnosis of acute abdominal pain: a multicentre study. BM Clin Res Ed 1986:293(6550):800-4.

9 Wraith SM, Aikins JS, Buchanan BG, et al. Computerized consultation system for selection of antimicrobial therapy. Am J Hosp Pharm 1976;33:1304-8.

10 Barness LA, Tunnessen WW Jr, Worley WE, et al. Computer-assisted diagnosis in pediatrics. Am J Dis Child 1974; 127:852-8.

11 Barnett GO, Cimino JJ, Hupp JA, et al. DXplain: an evolving diagnostic decision-support system. JAMA 1987;258:67-74.

12 Athreya BH, Cheh ML, Kingsland LC. Computer-assisted diagnosis of pediatric rheumatic diseases. Pediatrics 1998;102(4):E48.

13 Swender PT, Tunnessen WW Jr, Oski FA. Computer-assisted diagnosis. Am J Dis Child 1974:127:859-61.

14 Feldman M, Barnett GO. Pediatric computer-based diagnostic decision support. Scientific Program, Section on computers and other technologies. American Academy of Pediatrics, New Orleans, LA, October 27, 1991.

15 DeGroff CG, Bhatikar S, Hertzberg J, et al. Artificial neural network-based method of screening heart murmurs in children. Circulation 2001;103:2711-6.

16 Bosque EM. Symbiosis of nurse and machine through fuzzy logic: improved specificity of a neonatal pulse oximeter alarm. ANS Adv Nurs Sci 1995; 18:67-75.

17 Wyatt JC. Use and sources of medical knowledge. Lancet 1991;338:1368-73.

18 Ely JW, Osheroff JA, Ebell MH. Analysis of questions asked by family doctors regarding patient care. BM 1999;319(7206):358-61.

19 Vincent C, Neale M, Woloshynowych M. Adverse events in British hospitals: preliminary retrospective record review. $B M$ 2001;322(7285):517-19.

20 Alberti KG. Medical errors: a common problem. BM 2001;322(7285):501-2.

20a McKenna C. New online diagnostic tool launched to help doctors. BM 2002;324: 1478

21 Wyatt J, Spiegelhalter D. Field trials of medical decision-aids: potential problems and solutions. In: Clayton P, ed. Proceedings of the 15th Symposium on Computer Applications in Medical Care, Washington, 1991. New York: McGraw Hill, 1991:3-7.

22 McManus C. Engineering quality in health care. Qual Health Care 1996;5:127.

23 Purves I. Prodigy, a computer assisted prescribing scheme. BM 1996;313:1549.

24 Brahams D, Wyatt J. Decision-aids and the law. Lancet 1989;2:632-4.

25 Schoenberg R, Safran C. Internet based repository of medical records that retains patient confidentiality. BM 2000;321: 1199-203.

26 Rigby M, Forsström, Roberts R, et al. Verifying quality and safety in health informatics services. BM 2001;323:552-6. 